\title{
Application of white blood cell SPECT/CT to predict remission after a 6 or 12 week course of antibiotic treatment for diabetic foot osteomyelitis
}

\author{
Julien Vouillarmet $^{1} \cdot$ Myriam Moret $^{2} \cdot$ Isabelle Morelec $^{3} \cdot$ Paul Michon $^{1} \cdot$ \\ Julien Dubreuil $^{3}$
}

Received: 6 May 2017 / Accepted: 7 July 2017 / Published online: 2 September 2017

(C) Springer-Verlag GmbH Germany 2017

\begin{abstract}
Aims/hypothesis Diabetic foot osteomyelitis is a major risk factor for amputation. Medical treatment allows remission in $53-82 \%$ of cases. However, the optimal duration of antibiotic therapy remains controversial as a validated marker of osteomyelitis remission is lacking. The aim of this cohort study was to assess prospectively the remission rate of diabetic foot osteomyelitis medically treated using white blood cell (WBC)-single-photon emission computed tomography (SPECT)/computed tomography (CT) as a predictive marker of remission.

Methods Individuals with diabetic foot osteomyelitis that was non-surgically treated between April 2014 and December 2015 were included. All participants were treated with antibiotics alone. WBC-SPECT/CT was performed at 6 weeks and antibiotic treatment discontinued if the clinical signs of soft-tissue infection had resolved and there was no abnormal uptake of labelled WBCs. Treatment was otherwise continued for a total of 12 weeks and then discontinued. For these individuals, another WBC-SPECT/CT was performed at 12 weeks. Remission was defined as the absence of recurrence of osteomyelitis at the same location at 1 year.

Results Forty-five individuals were included; overall remission rate was $84 \%$ at 1 year. A 6 week course of antibiotics was used
\end{abstract}

Julien Vouillarmet

julien.vouillarmet@chu-lyon.fr

1 Hospices Civils de Lyon, Service d'Endocrinologie, Diabète et Obésité, Centre Hospitalier Lyon-Sud, 69495 Pierre Bénite, France

2 Hospices Civils de Lyon, Service d'Endocrinologie et Diabète, Groupement Hospitalier Est, Bron, France

3 Hospices Civils de Lyon, Service de Médecine Nucléaire, Centre Hospitalier Lyon-Sud, Pierre Bénite, France in 23 participants, 22 of whom were in remission at 1 year (96\%); a 12 week course was used for 22 participants, 16 of whom were in remission at 1 year $(73 \%)$. Sensitivity of WBCSPECT/CT at 12 weeks was $100 \%$, specificity $56 \%$, positive predictive value $46 \%$ and negative predictive value $100 \%$.

Conclusions/interpretation The study suggests that WBCSPECT/CT could predict remission at the end of antibiotic treatment.

Trial registration: ClinicalTrials.gov NCT02927678

Keywords Diabetic foot - Nuclear imaging - Osteomyelitis . Single photon emission computed tomography/computed tomography $\cdot$ Treatment

\begin{tabular}{|c|c|}
\hline \multicolumn{2}{|c|}{ Abbreviations } \\
\hline CRP & C-reactive protein \\
\hline $\mathrm{CT}$ & Computed tomography \\
\hline DFO & Diabetic foot osteomyelitis \\
\hline ESR & Erythrocyte sedimentation rate \\
\hline HMPAO & Hexamethylpropyleneamine oxime \\
\hline IDSA & Infectious Diseases Society of America \\
\hline IWGDF & $\begin{array}{l}\text { International Working Group on the } \\
\text { Diabetic Foot }\end{array}$ \\
\hline SPECT/CT & $\begin{array}{l}\text { Single photon emission computed } \\
\text { tomography }\end{array}$ \\
\hline $\mathrm{TcPO}_{2}$ & Transcutaneous oxygen pressure \\
\hline WBC & White blood cell \\
\hline
\end{tabular}

\section{Introduction}

Diabetic foot osteomyelitis (DFO) is a frequent and important concern for people with diabetes, in whom the prevalence is about $20 \%$ for those presenting with a mild diabetic foot 
infection and up to $60 \%$ for those hospitalised with severe infection [1]. Despite controversies in the management of DFO, the purely medical approach based on antibiotic therapy alone is efficient $[2,3]$. Indeed, the remission rate of DFO in those treated medically is reported to range from $53 \%$ to $82 \%$ [4-14].

Several factors are probably involved in the success of the medical treatment, including the choice of the appropriate antibiotics active against the bacterial strain located in the bone, the vascular status of the individual and the associated clinical details, such as absence of chronic kidney disease. Two other factors may also contribute to the variability in efficacy between studies. The first is the difficulty in predicting DFO remission; there is currently no clinical, biological or radiological factor predictive of remission to help physicians decide when to discontinue treatment. Some factors have been proposed for this (such as a decrease in previously elevated inflammatory markers, resolution of any underlying soft-tissue infection, healing of any wound, and radiographic changes that suggest healing), but without any strong supporting evidence in the literature [1]. The recent International Working Group on the Diabetic Foot (IWGDF) guidelines consider that the best way to define treatment success is the absence of infection recurrence at the initial site for 12 months after cessation of the treatment [15]. Some studies did not use a specific duration for evaluation (mainly because of a retrospective design) $[4-6,8,11]$ or used other variables, such as survival with limb intact [10] or ulcer healing $[7,11,13]$.

The second point is the controversy over the optimal duration of antibiotic treatment. When we planned the present study, most published work had treatment durations longer than 12 weeks for some or all of the individuals $[4,5,7-12]$. Based on these studies, the 2012 Infectious Diseases Society of America (IDSA), recommended more than 3 months' antibiotic treatment [1]. Tone et al in 2015 published the only randomised controlled study in this area, which compared 6 weeks with 12 weeks of treatment in 40 participants [14]. The remission rate was $60 \%$ in those treated for 6 weeks and $70 \%$ in those treated for 12 weeks. The difference was not statistically significant and the key message of the study was to recommend 6 weeks of antibiotic therapy. This was retained in the last IWGDF guidelines in 2016 [15]. Studies to investigate the optimal duration of antibiotic treatment in DFO and to determine when DFO has been arrested after treatment were requested by IDSA experts to improve management [1] Long-term use of antibiotics is associated with side effects such as acute kidney injury and also bacterial resistance, and thus a test or imaging method to assess the need for antibiotics would help clinicians.

Our retrospective study of individuals with a wide range of antibiotic treatment durations showed that a negative white blood cell (WBC)-single photon emission computed tomography (SPECT)/computed tomography (CT) could be an effective predictive marker of osteomyelitis remission at the end of antibiotic treatment [12]. This study examines the potential of WBC-SPECT/CT to predict remission after a 6 or 12 week course of antibiotic treatment for DFO.

\section{Methods}

Study design We prospectively assessed DFO remission at 1 year after completion of the antibiotic treatment in participants with non-surgically treated DFO. The duration of antibiotic treatment was predefined according to clinical findings (soft-tissue inflammation) and the results of WBC-SPECT/CT performed after 6 weeks of antibiotic treatment. If there was no sign of soft-tissue infection, and the examination was interpreted as negative, antibiotics were discontinued. If there was persistent soft-tissue infection and/or positive WBCSPECT/CT (defined by an abnormal uptake of labelled WBCs), antibiotics were continued for a further 6 week cycle and then discontinued for all participants. Another WBCSPECT/CT was performed at 12 weeks. Participants without either clinical signs of inflammation or a positive SPECT/CT (at 6 or 12 weeks) were followed until ulcer healing and/or 1 year after antibiotic discontinuation. Participants with a positive WBC-SPECT/CT at 12 weeks were followed monthly with clinical and/or radiological assessment until relapse (recurrent bone infection at the initial site) or for at least 1 year. In the case of relapse, a medical and/or surgical treatment was instituted.

Population Consecutive individuals with DFO seen in an outpatient setting or during hospitalisation were prospectively included in two diabetic foot centres in Lyon, France, between April 2014 and December 2015. Those with an indication for bone resection, or minor or major amputation, were not included, nor were those with a contraindication to WBC-SPECT/CT or who refused the WBC-SPECT/CT. Individuals with peripheral arteriopathy or renal insufficiency were not excluded in the absence of an indication for bone resection or amputation. As in recent randomised controlled studies on DFO [13, 14], at least 40 participants were included, with a minimum of 20 participants treated for 6 weeks and 20 participants treated for 12 weeks, with an expected rate of DFO remission above $70 \%$.

The investigations were carried out in accordance with the principles of the Declaration of Helsinki. The study was registered with the institutional office of the national data protection agency (Commission Nationale de l'Informatique et des Libertés; ID: 15-010) and registered on ClinicalTrials.gov (NCT02927678). In accordance with legislation in place in France at the time of the study, ethics approval was not required, and oral informed consent was collected for all participants. 
Outcome assessment DFO was defined in accordance with IWGDF guidelines by the association of soft-tissue infection and/or positive probe-to-bone test, and signs suggestive of osteomyelitis on plain radiographs (cortical disruption, periosteal elevation, a sequestrum or involucrum, or gross bone destruction) [15]. Systematic bone biopsy was not performed. The primary outcome was overall remission of DFO, defined by the absence of recurrent infection at the initial site and the absence of need for surgical bone resection or amputation at least 12 months after completion of antibiotic treatment [15]. Relapse was defined as a recurrent bone infection at the initial site. The secondary outcomes included remission at least 12 months after completion of antibiotic treatment in those treated for 6 weeks, and the diagnostic qualities of WBCSPECT/CT at the end of antibiotic treatment to determine remission (sensitivity, specificity, positive and negative predictive values for those treated for 12 weeks, and negative predictive value for those treated for 6 weeks). None of the participants with a negative SPECT/CT during the study had another examination at follow-up to observe a potential positive test result.

Medical treatment Individuals were treated according to guidelines [1, 15]: first, with an empirical antibiotic therapy based on infection severity, and second with treatment according to the results of microbiological culture. Antibiotic administration was intravenous, oral or intravenous followed by oral. The type of antibiotic was left to clinical judgement, taking into account allergies and comorbidities (notably renal function). A combination of at least two antibiotics was recommended. Deep wound samples were collected after debridement and cleansing of the ulcer with sterile $\mathrm{NaCl}$ solution. Bone biopsy was not routinely performed. All physicians adopted the same approach to wound care, including daily dressing and offloading of the ulcer. The duration of antibiotic treatment was predefined, as described above.

Variables Information on diabetes history and chronic complications was collected, as well as characteristics of the diabetic foot ulcer. Healing was defined by complete epithelialisation of the wound. Vascular assessment was performed at baseline and included pressure index measurement and transcutaneous partial oxygen pressure. Peripheral arteriopathy was defined by an ankle pressure index $<0.9$ and/or a toe systolic pressure $<70 \mathrm{mmHg}$ and/or a transcutaneous oxygen pressure $\left(\mathrm{TcPO}_{2}\right)$ measurement $<30 \mathrm{mmHg}$. Blood samples were collected at baseline and after 6 and/or 12 weeks of antibiotic treatment to measure C-reactive protein (CRP), neutrophil count and creatininaemia. Nephropathy was defined by the presence of at least one of the following

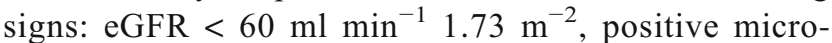
albuminuria or positive proteinuria. All participants had radiographs centred on the site of infection at the initial visit, at the end of antibiotic treatment and at 1 year.

WBC-SPECT/CT imaging Blood samples (42 ml) were collected on citric acid dextrose to label WBCs with $99 \mathrm{mTc}$ hexamethylpropyleneamine oxime (HMPAO). Cell-rich plasma was obtained after sedimentation for $30-90 \mathrm{~min}$ at $37^{\circ} \mathrm{C}$ in the presence of $6.5 \mathrm{ml}$ heafusine. Granulocytes were labelled with $650 \mathrm{MBq}$ of freshly prepared 99mTc-HMPAO (Ceretec; Amersham, Mississauga, ON, Canada); incubation was for $15 \mathrm{~min}$ at room temperature $\left(20^{\circ} \mathrm{C}\right)$. The labelled cells were washed and suspended in cell-poor plasma, then re-injected intravenously with a delay of no more than $3 \mathrm{~h}$ after collection of the initial blood sample.

Scintigraphic images were acquired $2 \mathrm{~h}$ and $20 \mathrm{~h}$ after injection of the 99mTc-HMPAO-labelled cells with a gamma camera (Philips Skylight; Philips, Amsterdam, the Netherlands). An incidence focus on the feet (a $256 \times 256$ matrix, pre-time $=10 \mathrm{~min}$ at $2 \mathrm{~h}$ and $15 \mathrm{~min}$ at $20 \mathrm{~h}$ ) was used (plantar with zoom and lateral views). A hybrid SPECT/CT system (Siemens Symbia T2; Siemens, Munich, Germany) was used for imaging at $20 \mathrm{~h}$. Interpretation of WBCSPECT/CT was based on uptake of labelled WBCs, on recruitment between $2 \mathrm{~h}$ and $24 \mathrm{~h}$ acquisitions, and with the proximity with bone on SPECT/CT. All examinations were interpreted prospectively as positive or negative by a nuclear physician informed of the initial site of the DFO and with over 20 years' experience of WBC-SPECT/CT.

Statistical analysis Continuous variables were described using mean and SD; categorical variables were described using group size and percentage. Continuous variables were compared using a two-sample $t$ test and categorical variables with Fisher's exact test. A $p$ value $<0.05$ was fixed as the threshold of statistical significance. Analyses were performed using Prism software version 5.0c (GraphPad Software, La Jolla, CA, USA).

\section{Results}

Population Of the 98 individuals identified as eligible, 45 were included in the study. Of the 53 who did not join, 30 had an episode of DFO with an indication for bone resection, minor or major amputation (11 with necrosis and/or severe arteriopathy, 11 with important tissue loss, four with important foot deformities, two with poor general health, one uncontrolled sepsis and one severe adverse reaction) and a further 23 had a contraindication for scintigraphy (two dementia, one non-adherent, one difficult venous access) or refused to undergo this procedure $(n=19)$. One patient presented with a second DFO episode at another site after a relapse and only the 
first episode was included. All participants were followed for 1 year after completion of the antibiotic treatment.

Descriptive data The main characteristics of the participants are summarised in Table 1. Of the 45 participants, $82 \%(n=37)$ required hospitalisation, the mean \pm SD duration of which was $15 \pm 9$ days. All but one individual had a wound at baseline. Osteomyelitis was located on the toes in 19 participants (42\%), the metatarsal bone in 25 participants $(56 \%)$, and on the rear foot in one participant (2\%). A total of 14 participants had received antibiotic treatment before inclusion: $4 / 23(17 \%)$ in the group treated for 6 weeks and 10/22 (45\%) in the group treated for 12 weeks $(p=0.21)$. All but one were treated orally.

The pathogens identified in deep wound samples are presented in Table 2. Methicillin-resistant $S$. aureus was not observed. Blood culture was positive for five participants (11\%). An intravenous antibiotic regimen was used as first-line treatment in $38 \%(n=17)$ of the participants, the mean \pm SD duration of which was $14 \pm 12$ days, followed by a course of oral antimicrobial therapy. Regarding mode of treatment, $13 \%$ $(n=6)$ were treated exclusively with intravenous broadspectrum $\beta$-lactams and/or glycopeptides and 49\% $(n=22)$ exclusively with an oral antibiotic regimen. Antibiotics were used in combination; the most frequent antibiotic used was ofloxacin $(82 \%, n=37)$, followed by amoxicillin/clavulanic

Table 1 Baseline characteristics of the population $(n=45)$

\begin{tabular}{|c|c|}
\hline Characteristic & $n(\%) /$ mean $\pm \mathrm{SD}$ \\
\hline Age (years) & $64 \pm 11$ \\
\hline Male sex & $37(80)$ \\
\hline Diabetes duration (years) & $18 \pm 11$ \\
\hline Type 2 diabetes & $40(87)$ \\
\hline $\mathrm{HbA}_{1 \mathrm{c}}(\%)$ & $8.3 \pm 1.9$ \\
\hline $\mathrm{HbA}_{1 \mathrm{c}}(\mathrm{mmol} / \mathrm{mol})$ & $67 \pm 9$ \\
\hline Neuropathy & $42(91)$ \\
\hline Retinopathy & $28(62)$ \\
\hline Nephropathy $^{\mathrm{a}}$ & $29(64)$ \\
\hline $\mathrm{eGFR}<60 \mathrm{ml} \mathrm{min}^{-1} 1.73 \mathrm{~m}^{-2}$ & $13(29)$ \\
\hline Peripheral arteriopathy ${ }^{\mathrm{b}}$ & $12(27)$ \\
\hline Cardiovascular disease & $15(33)$ \\
\hline \multicolumn{2}{|l|}{ Diabetes treatment } \\
\hline OAD or incretin & $20(43)$ \\
\hline OAD or incretin-insulin & $10(22)$ \\
\hline Insulin & $16(35)$ \\
\hline History of osteomyelitis & $18(39)$ \\
\hline History of amputation & $16(35)$ \\
\hline
\end{tabular}

${ }^{\mathrm{a}}$ Defined by the presence of at least one of: eGFR $<60 \mathrm{ml} \mathrm{min}^{-1}$ $1.73 \mathrm{~m}^{-2}$, positive microalbuminuria or positive proteinuria

${ }^{\mathrm{b}}$ Defined by an ankle pressure index $<0.9$ and/or a toe systolic pressure $<70 \mathrm{mmHg}$ and/or $\mathrm{TcPO}_{2}<30 \mathrm{mmHg}$

$\mathrm{OAD}$, oral glucose-lowering drug acid $(49 \%, n=22)$, trimethoprim-sulfamethoxazole $(15 \%$, $n=7)$ and pristinamycin $(13 \%, n=6)$; other antibiotics were used in five or fewer episodes each.

The overlying soft-tissue wound was healed at the end of antibiotic treatment (either 6 or 12 weeks) in $49 \%(n=22)$ of participants; a reduction in wound area $>50 \%$ or healing was observed in $75 \%$. The mean \pm SD time to healing at the end of antibiotic treatment was $14.1 \pm 9$ weeks; it was $11.4 \pm 6.8$ for individuals treated for 6 weeks, and $17.1 \pm 10.2$ for those treated for 12 weeks $(p<0.01)$. Non-healing of the wound at the end of follow-up was observed in five participants, including one participant in remission.

A 6 week course of antibiotics was used for 23 participants $(51 \%)$, and a 12 week course for 22 participants (49\%) according to the results of WBC-SPECT/CT and clinical findings (Fig. 1). One patient had persistent signs of soft-tissue inflammation at 6 weeks, with abnormal WBC-SPECT/CT, and therefore antibiotic treatment was extended to 12 weeks. Wound, infectious and vascular variables at baseline, 6 and 12 weeks according to the duration of antibiotic treatment are presented in Table 3 . There were no statistically significant differences between the two groups at baseline for wound duration before DFO, wound size, presence of cellulitis, fever, positive probe-to-bone test, CRP level or the presence of peripheral arteriopathy. At 6 weeks, there were no differences between the two groups for wound size, reduction of wound area $>50 \%$ or healing, neutrophils or CRP level. Participants treated for 12 weeks had a significantly higher level of neutrophils $(p=0.04)$ and intravenous antibiotic treatment was more frequently used $(p<0.01)$ at baseline but also a significantly higher rate of persistent positive probe-to-bone test at 6 weeks $(p=0.04)$ compared with those treated for 6 weeks.

Outcome data At 1 year after completion of antibiotic treatment, remission of DFO was observed in $84 \%(n=38)$ of the participants (Fig. 1). A relapse occurred in seven individuals after a median (range) of 5 months (1-10 months); at the end of treatment, 6/7 had an abnormal WBC-SPECT/CT (Fig. 2), 6/7 had a persistent wound, 2/7 a positive probe-to-bone test, the mean $\pm \mathrm{SD}$ level of neutrophils was $3.44 \pm 0.44 \times 10^{9} / 1$, and that of CRP was $57 \pm 29 \mathrm{nmol} / \mathrm{l}$. Relapses were managed by minor amputation in five participants and by second-line antibiotic treatment in two individuals. Remission of DFO was $96 \%$ $(n=22)$ in participants treated for 6 weeks and $73 \%(n=16)$ in those treated for 12 weeks. The presence of peripheral arteriopathy and renal insufficiency did not impact on remission of DFO; the remission rates were $91.7 \%$ and $100 \%$, respectively.

For those treated for 6 weeks, the negative predictive value of WBC-SPECT/CT at the end of treatment was 96\%. For those treated for 12 weeks, the sensitivity of WBC-SPECT/ CT at the end of treatment was $100 \%$, specificity was $56 \%$, positive predictive value was $46 \%$ and the negative predictive value was $100 \%$. 
Table 2 Distribution of pathogens identified from deep wound samples

\begin{tabular}{lccc}
\hline & \multicolumn{2}{l}{ Incidence } & \\
\cline { 2 - 4 } Pathogen & Total $(n=103)$ & 6 weeks $(56$ cases $)$ & 12 weeks (47 cases) \\
\hline Gram-positive cocci & $54(52)$ & $27(48)$ & $27(57)$ \\
Methicillin-sensitive S. aureus & $24(23)$ & $14(25)$ & $10(21)$ \\
Coagulase-negative staphylococci & $5(5)$ & $1(2)$ & $4(9)$ \\
Streptococcus spp. & $17(16)$ & $7(12)$ & $10(21)$ \\
Enterococcus faecalis & $5(5)$ & $4(7)$ & $1(2)$ \\
Enterobacter cloacae & $3(3)$ & $1(2)$ & $2(4)$ \\
Gram-negative bacilli & $34(33)$ & $23(41)$ & $3(23)$ \\
Proteus spp. & $10(10)$ & $7(12)$ & $3(6)$ \\
Morganella morganii & $6(6)$ & $3(5)$ & $1(2)$ \\
Pseudomonas aeruginosa & $5(5)$ & $4(7)$ & $2(4)$ \\
Klebsiella spp. & $6(6)$ & $4(7)$ & $2(4)$ \\
Other & $7(7)$ & $5(9)$ & $6(13)$ \\
Obligate anaerobes & $10(10)$ & $4(7)$ & $3(6)$ \\
Gram-positive bacilli & $4(4)$ & $1(2)$ & $0(0)$ \\
Gram-negative cocci & $1(1)$ & $1(2)$ & \\
\hline
\end{tabular}

Data are $n(\%)$

\section{Discussion}

The study suggests that WBC-SPECT/CT predicts remission after a 6 or 12 week course of antibiotic treatment, and found that the overall remission rate was higher than most of the rates observed in the literature [4-14]. As is generally observed in studies dealing with DFO, the overwhelming majority of the population included in the present study had neuropathy and there was a high rate of chronic complications associated with long-term diabetes. However, contrary to recent studies [13,14], the population included individuals with peripheral arteriopathy and one-quarter had renal

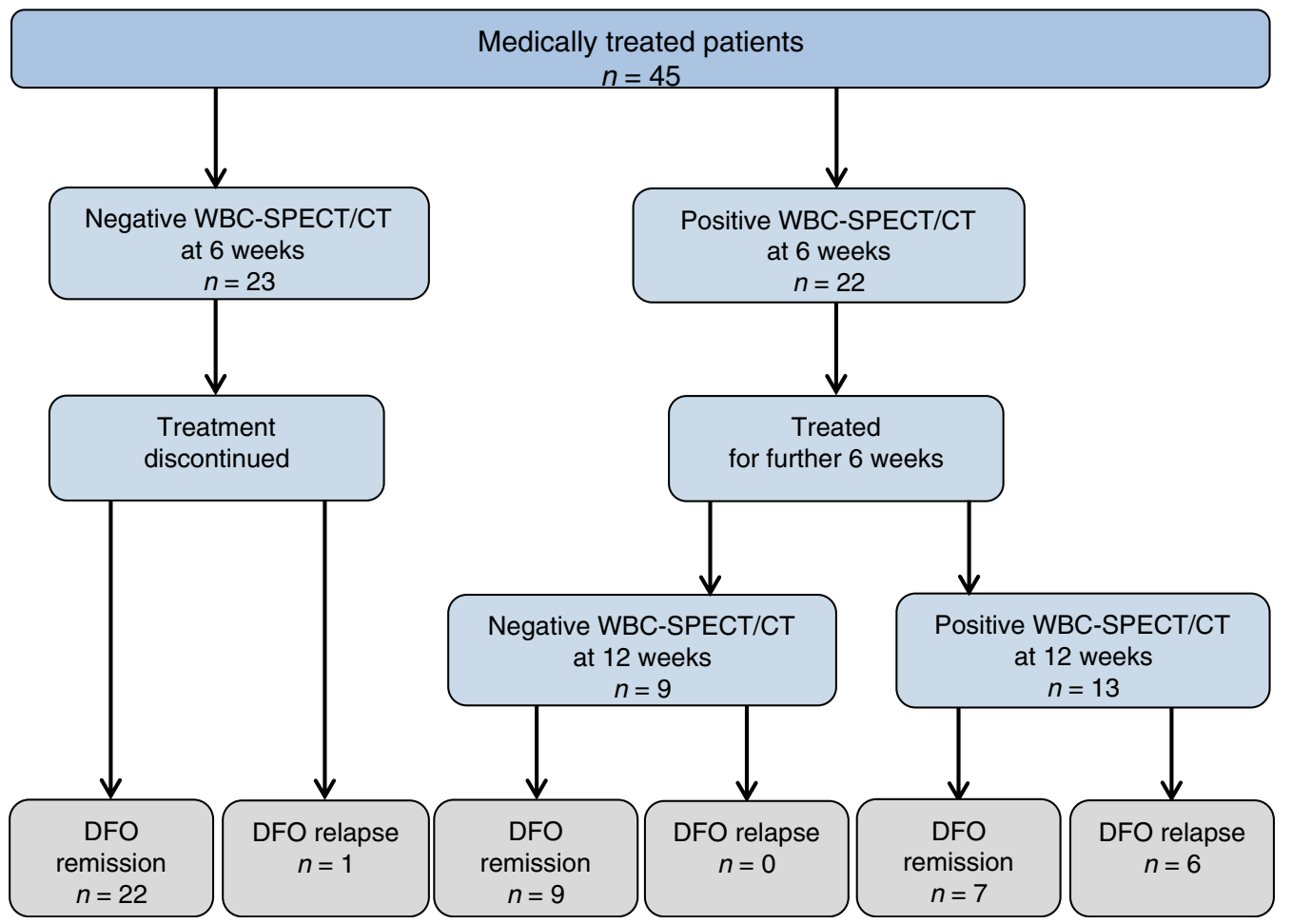

Fig. 1 Flow of participants through the study. Relapse was classed as infection at the initial site. Remission was the absence of relapse after 1 year of follow-up following antibiotic discontinuation 
Table 3 Wound, infectious and vascular variables according to the duration of antibiotic treatment

\begin{tabular}{|c|c|c|c|c|c|}
\hline \multirow[t]{2}{*}{ Variable } & \multicolumn{2}{|c|}{6 weeks' treatment $(n=23)$} & \multicolumn{3}{|c|}{12 weeks' treatment $(n=22)$} \\
\hline & Baseline & 6 weeks & Baseline & 6 weeks & 12 weeks \\
\hline Wound duration (weeks) ${ }^{\mathrm{a}}$ & $19.7 \pm 36.9$ & NA & $15.7 \pm 25.7$ & NA & NA \\
\hline Wound size $\left(\mathrm{mm}^{2}\right)^{\mathrm{a}}$ & $124 \pm 117$ & $77 \pm 120$ & $226 \pm 240$ & $122 \pm 127$ & $110 \pm 165$ \\
\hline Wound healing $^{\mathrm{a}}$ & 0 & $15(65)$ & $1(5)$ & $2(9)$ & $7(32)$ \\
\hline Healing or reduction wound area $>50 \%{ }^{\mathrm{a}}$ & NA & $19(83)$ & NA & $14(64)$ & $15(68)$ \\
\hline Probe-to-bone test & $17(74)$ & $0 *$ & $13(59)$ & $5(23) *$ & $3(14)$ \\
\hline Cellulitis $>2 \mathrm{~cm}$ & $18(78)$ & 0 & $17(77)$ & $1(5)$ & $1(5)$ \\
\hline Fever $(\%)$ & $2(9)$ & 0 & $6(27)$ & 0 & 0 \\
\hline Neutrophils $\left(\mathrm{X} 10^{9} / 1\right)$ & $5.54 \pm 2.01^{*}$ & $3.67 \pm 1.33$ & $7.03 \pm 2.88^{*}$ & $3.61 \pm 1.57$ & $3.79 \pm 1.42$ \\
\hline CRP (nmol/l) & $724 \pm 686$ & $67 \pm 143$ & $867 \pm 771$ & $57 \pm 57$ & $48 \pm 29$ \\
\hline Peripheral arteriopathy ${ }^{\mathrm{b}}$ & $8(35)$ & $\mathrm{NC}$ & $4(18)$ & $\mathrm{NC}$ & $\mathrm{NC}$ \\
\hline Previous antibiotic therapy & $4(17)$ & NA & $10(45)$ & NA & NA \\
\hline Initial parenteral antibiotic therapy & $7(30)^{*}$ & NA & $18(82)^{*}$ & NA & NA \\
\hline
\end{tabular}

Data are $n(\%)$ or mean $\pm \mathrm{SD}$

${ }^{\mathrm{a}}$ one participant treated for 12 weeks did not have a wound at baseline

${ }^{\mathrm{b}}$ Defined by an ankle pressure index $<0.9$ and/or a toe systolic pressure $<70 \mathrm{mmHg}$ and/or $\mathrm{TcPO}_{2}<30 \mathrm{mmHg}$

${ }^{*} p<0.05$ between episodes in those treated for 6 weeks and those treated for 12 weeks

NA, not applicable, NC, not collected

insufficiency; although both are linked with poor prognosis, their prevalence does not seem to have negatively affected the rate of DFO remission at 12 months after completion of antibiotic treatment.
Fig. 2 WBC-SPECT/CT after 12 weeks of treatment. There is a significant accumulation of leucocytes at the right fifth metatarsal bone $2 \mathrm{~h}$ after injection (arrow) (a), with recruitment at $20 \mathrm{~h}$ (arrow) (b) extending to the cortex of the bone and causing cortical erosion on SPECT-CT (arrows) (c-e). This individual relapsed at 4 months after the end of treatment
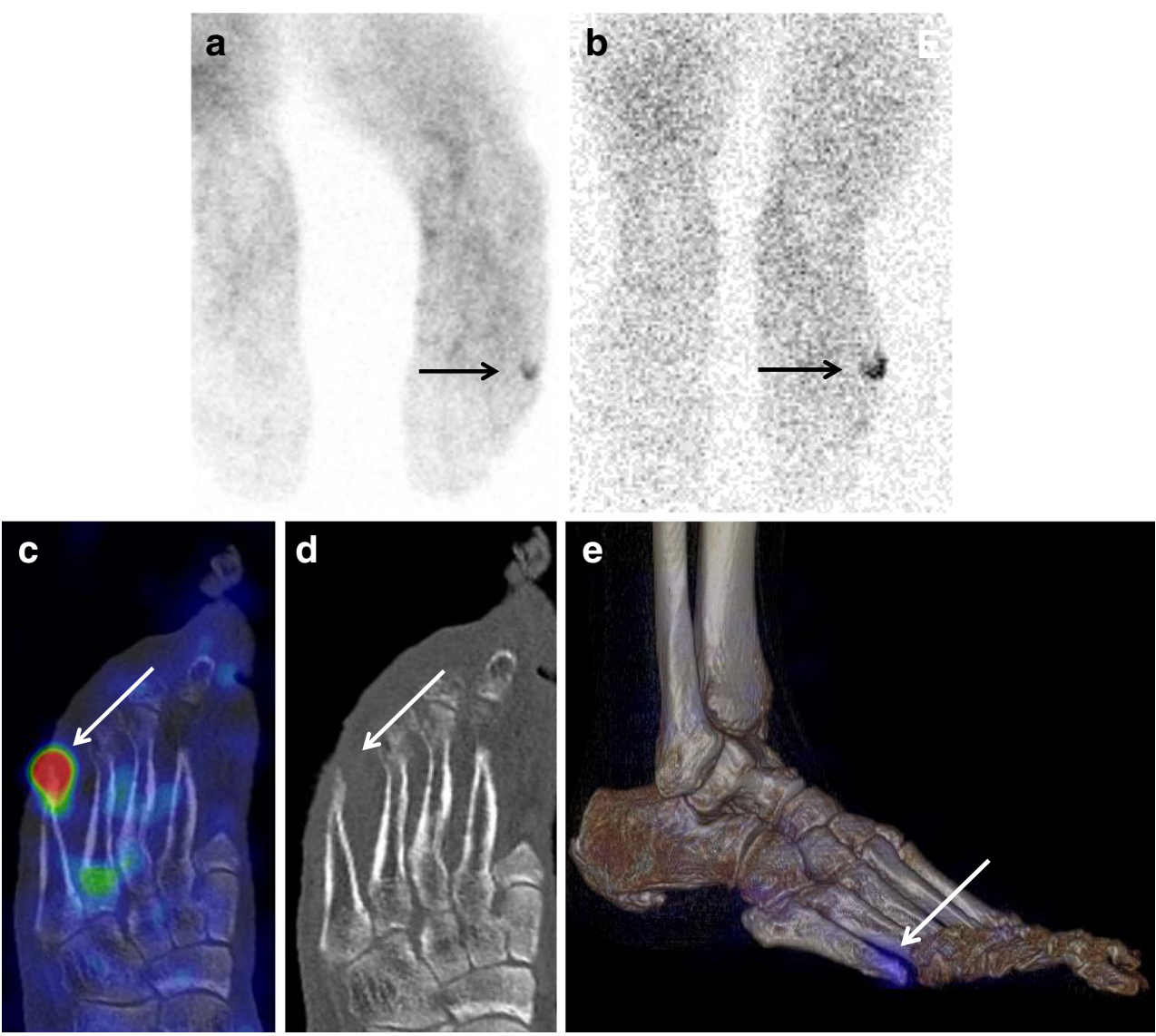
There are few reported data dealing with the duration of antibiotic treatment in DFO. The paucity of data contrasts with the importance of this question, as the duration of antibiotic treatment is associated with the frequency of side effects and the overall cost of care [14]. At the time we planned the study, and on the basis of data available in the literature, guidelines recommended at least a 12 week course of antibiotic treatment in the absence of surgery [1]. We hypothesised that a shorter 6 week course may be sufficient for some individuals and thus used this period of treatment in the study design. Interestingly, the study by Tone et al [14] was subsequently published and the IWGDF guidelines were modified to recommend a 6 week course of antibiotic treatment [15]. However, as mentioned by Tone et al in the discussion of their study, the small population size could have induced a high risk of beta error, leading them to conclude that a difference was absent when, in fact, it existed [14]. Furthermore, the authors did not find any factor predictive for remission, and thus it cannot be excluded with certainty that some individuals may benefit from treatment for longer than 6 weeks.

The IWGDF also underline that the question of the duration of antibiotic therapy to treat DFO remains controversial [15]. The difficulty in choosing the optimal duration of antibiotic treatment relates to the absence of a validated tool to predict DFO remission. Guidelines suggest different markers that could be helpful to predict the efficiency of medical treatment in DFO: radiographical changes, healing of any wound, decrease in previously elevated inflammatory markers and resolution of any underlying soft-tissue infection [1]. However, evidence to support these recommendations is poor. For instance, the reproducibility [16] and diagnostic characteristics [12] of radiological examinations to predict remission are too poor for application in routine clinical care. More generally, healing of any wound is clearly an important factor to prevent any new foot infection, but it is of note that, although all but one individual with relapse in our study had a non-healed wound at the end of treatment, not all of those who had a non-healed wound had relapse. Thus, wound healing may not be used exclusively to assess the remission of DFO, as it has been in some studies $[7,11,13]$. Factors other than infection affect the healing process, such as the size of the ulcer at baseline, compliance with off-loading, tissue perfusion, nutritional status and so on [17]. Some studies suggest a predictive role for erythrocyte sedimentation rate (ESR) [18-20] and CRP trajectories when monitoring DFO treatment $[19,20]$. Yet none of these studies assessed the remission rate of DFO according to the level of these markers of inflammation at the end of treatment. In this study, CRP level at baseline and during follow-up was not associated with DFO remission. As ESR varies according to many factors (e.g. age, sex, anaemia, obesity), this variable was not routinely measured in the participating centres and so was not collected in this study. It cannot be formally excluded that ESR, which normalises more slowly than other inflammatory markers, could be more valuable [18-20].

The last point is the remission of any underlying soft-tissue infection. This factor was included in our decision to discontinue antibiotic treatment at 6 weeks. Interestingly, only one participant had a persistent soft-tissue inflammation at 6 weeks, and this person also had an abnormal WBCSPECT/CT at 6 weeks. This suggests that persistence of soft-tissue inflammation alone may have a good positive predictive value but a low sensitivity.

Animal and human studies have shown that nuclear imaging could be of interest to predict osteomyelitis remission [21-24]. However, these studies used isotopes with a high radiation burden and low spatial resolution. Lipsky later suggested that a leucocyte scan might be useful in demonstrating that infection has been arrested [25]. We published in $2014 \mathrm{a}$ paper suggesting that WBC-SPECT/CT could be used to predict DFO remission at the end of medical treatment with an optimal negative predictive value $(100 \%)$ and that it could therefore be a very useful tool to guide the duration of antibiotic treatment in DFO [12]. The interest in WBC-SPECT/CT to assess DFO remission was also confirmed by Lazaga et al, who found a negative predictive value of $83 \%$ [26]. This was further confirmed for those treated for 6 weeks in the present study. The limited value of this examination is, however, related to the high rate of false positives, with positive predictive values ranging from $50 \%$ [26] to $70 \%$ [12]; a high false-positive rate could lead to overtreatment. For instance, in the current study some individuals who were treated for 12 weeks may have had the same outcomes if they had been treated for 6 weeks.

WBC-SPECT/CT seems to better select individuals who need only a 6 week course of treatment: all but one participant treated for 6 weeks in our study was in remission at least 12 months after completion of antibiotic treatment, which is far from the $60 \%$ observed by Tone et al [14], although comparing studies is difficult. Conversely, this technique could also help to select individuals who need a longer course of antibiotic treatment, and it is of note that such participants were probably more severely ill than those treated for 6 weeks (as evidenced by a higher neutrophil count and a more frequent use of intravenous antibiotic treatment at baseline). However, WBC-SPECT/CT is costly, not widely available and also time consuming, and so cannot be recommended for routine care. It could be proposed that WBC-SPECT/CT be performed at 6 weeks of treatment in a subset of individuals (for example, those with a persistent wound, probe-to-bone test, soft-tissue inflammation and/or elevated inflammatory marker), but this approach needs to be confirmed in studies. It is also of note that, in addition to WBC-SPECT/CT, other recent nuclear imaging techniques appear to be promising for the diagnosis and management of $\mathrm{DFO}$, though none is currently validated to predict DFO remission [27-31]. 
The study has some limitations that are important to discuss. First, bone biopsy was not performed to confirm diagnosis of DFO. However, the most recent IWGDF guidelines recommend a bone biopsy only when the diagnosis is in doubt or determining the antibiotic susceptibility is crucial [15]. It is important to note that, even for research purposes, bone biopsy is not commonly performed [4-6, 10-13]; only one retrospective study in the literature has assessed its capacity to improve DFO remission [9]. Moreover, bone biopsy, through sampling errors, may lead to false-negative microbiological culture results. Indeed, even in trained teams, the rate of negative bone culture despite suspicion of DFO is reported to be around 20\% [14]. More generally, in the absence of bone specimen, we cannot be sure to target specifically the bacterial specimen located in the bone based only on deep wound samples $[32,33]$. It remains possible that participants in our study who had a relapse received an antibiotic regimen not adapted to the bacterial species in the bone, which is a well-known factor for failure [9]. Conversely, some of the participants were treated exclusively by broad-spectrum $\beta$-lactams and/or glycopeptides and it is possible that a bone biopsy could have helped to narrow the spectrum used. It can be noticed that the use of rifampicin was rare (three participants, data not shown), following recommendations to limit the development of additional bacterial resistance [9] in the absence of bone biopsy. It is important to note that, despite the absence of WBCSPECT/CT at baseline, differential diagnoses such as active Charcot disease, recent trauma or non-septic arthritis were unlikely. Charcot disease usually presents with moderate widespread uptake without any WBC recruitment at $20 \mathrm{~h}$ [34]. Recent trauma could involve recruitment of WBC owing to debridement by neutrophils and macrophages, but the fusion images with thin CT slices often allow diagnosis $[35,36]$. It is also possible to differentiate non-septic arthritis from septic forms as the latter present WBC recruitment at $20 \mathrm{~h}$ [37]. Furthermore, the physician who interpreted the data (IM) has 20 years' experience in this field.

To conclude, the study suggests that WBC-SPECT/CT could predict remission at the end of the antibiotic treatment, but these results need to be confirmed in other centres and with larger numbers of people.

Acknowledgements We thank P. Robinson (DRCI-Hospices Civils de Lyon) for help with manuscript preparation.

Funding This research received no specific grant from any funding agency in the public, commercial or not-for-profit sectors.

Data availability The datasets generated and/or analysed during the current study are available from the corresponding author on reasonable request.

Duality of interest The authors declare that there is no duality of interest associated with this manuscript.
Contribution statement JV conceptualised and designed the study, contributed to data analysis and interpretation, and wrote the manuscript. MM, PM, IM and JD contributed to the acquisition, analysis and interpretation of the data. All authors were involved in revising the manuscript critically for important intellectual content and for final approval of the version to be published. JV is the guarantor of this work and, as such, had full access to all the data in the study and takes responsibility for the integrity of the data and the accuracy of the data analysis.

\section{References}

1. Lipsky BA, Berendt AR, Cornia PB et al (2012) 2012 Infectious Diseases Society of America clinical practice guideline for the diagnosis and treatment of diabetic foot infections. Clin Infect Dis 54: 132-173

2. Jeffcoate WJ, Lipsky BA (2004) Controversies in diagnosing and managing osteomyelitis of the foot in diabetes. Clin Infect Dis 39: $115-122$

3. Game FL (2010) Management of osteomyelitis of the foot in diabetes mellitus. Nat Rev Endocrinol 6:43-47

4. Bamberger DM, Daus GP, Gerding DN (1987) Osteomyelitis in the feet of diabetic patients: long-term results, prognostic factors and the role of antimicrobial and surgical therapy. Am J Med 83:653-660

5. Venkatesan P, Lawn S, Macfarlane RM, Fletcher EM, Finch RG, Jeffcoate W (1997) Conservative management of osteomyelitis in the feet of diabetic patients. Diabet Med 14:487-490

6. Pittet D, Wyssa B, Herter-Clavel C, Kursteiner K, Vaucher J, Lew DP (1999) Outcome of diabetic foot infections treated conservatively: a retrospective cohort study with long-term follow-up. Arch Intern Med 159:851-856

7. Senneville E, Yazdanpanah Y, Cazaubiel M et al (2001) Rifampicin-ofloxacin oral regimen for the treatment of mild to moderate diabetic foot osteomyelitis. J Antimicrob Chemother 48:927-930

8. Embil JM, Rose G, Trepman E et al (2006) Oral antimicrobial therapy for diabetic foot osteomyelitis. Foot Ankle Int 27:771-779

9. Senneville E, Lombart A, Beltrand E et al (2008) Outcome of diabetic foot osteomyelitis treated non-surgically: a retrospective cohort study. Diabetes Care 31:637-642

10. Game FL, Jeffcoate WJ (2008) Primarily non-surgical management of osteomyelitis of the foot in diabetes. Diabetologia 51:962-967

11. Valabhji J, Oliver N, Samarasinghe D, Mali T, Gibbs RG, Gedroyc WM (2009) Conservative management of diabetic forefoot ulceration complicated by underlying osteomyelitis: the benefits of magnetic resonance imaging. Diabet Med 26:1127-1134

12. Vouillarmet J, Morelec I, Thivolet C (2014) Assessing diabetic foot osteomyelitis remission with white blood cell SPECT/CT imaging. Diabet Med 31:1093-1099

13. Lázaro-Martínez JL, Aragón-Sánchez J, García-Morales E (2014) Antibiotics versus conservative surgery for treating diabetic foot osteomyelitis: a randomized comparative trial. Diabetes Care 37: 789-795

14. Tone A, Nguyen S, Devemy F et al (2015) Six-week versus twelveweek antibiotic therapy for nonsurgically treated diabetic foot osteomyelitis: a multicenter open-label controlled randomized study. Diabetes Care 38:302-307

15. Lipsky BA, Aragón-Sánchez J, Diggle M et al (2016) IWGDF guidance on the diagnosis and management of foot infections in persons with diabetes. Diabetes Metab Res Rev 32:45-74

16. Álvaro-Afonso FJ, Lázaro-Martínez JL, Aragón-Sánchez J, GarcíaMorales E, Cecilia-Matilla A, Beneit-Montesinos JV (2013) Interobserver and intraobserver reproducibility of plain X-rays in 
the diagnosis of diabetic foot osteomyelitis. Int J Low Extrem Wounds 12:12-15

17. Game FL, Attinger C, Hartemann A et al (2016) IWGDF guidance on use of interventions to enhance the healing of chronic ulcers of the foot in diabetes. Diabetes Metab Res Rev 32:75-83

18. Michail M, Jude E, Liaskos C et al (2013) The performance of serum inflammatory markers for the diagnosis and follow-up of patients with osteomyelitis. Int J Low Extrem Wounds 12:94-99

19. Van Asten SA, Nichols A, La Fontaine J, Bhavan K, Peters EJ, Lavery LA (2017) The value of inflammatory markers to diagnose and monitor diabetic foot osteomyelitis. Int Wound J 14:40-45

20. Van Asten SA, Jupiter DC, Mithani M, La Fontaine J, Davis KE, Lavery LA (2017) Erythrocyte sedimentation rate and C-reactive protein to monitor treatment outcomes in diabetic foot osteomyelitis. Int Wound J 14:142-148

21. Graham GD, Lundy MM, Frederick RJ, Berger DE, O’Brien AW, Brown TJ (1983) Predicting the cure of osteomyelitis under treatment: concise communication. Nucl Med 24:110-113

22. McCarthy K, Velchik MG, Alavi A, Mandell GA, Esterhai JL, Goll S (1988) Indium-111-labeled white blood cells in the detection of osteomyelitis complicated by a pre-existing condition. J Nucl Med 29:1015-1021

23. Van der Wall H, Lunz D, Stanton F, Bruce W (2001) Prognostic value of $99 \mathrm{mTc}$ leukocyte scintigraphy in diabetic pedal osteomyelitis. Foot Ankle Int 22:720-724

24. Newman LG, Waller J, Palestro CJ et al (1991) Unsuspected osteomyelitis in diabetic foot ulcers. Diagnosis and monitoring by leukocyte scanning with indium in 111 oxyquinoline. J Am Med Assoc 266:1246-1251

25. Lipsky BA (2004) Medical treatment of the infected diabetic foot. Clin Infect Dis 39:S104-S114

26. Lazaga F, Van Asten SA, Nichols A et al (2016) Hybrid imaging with $99 \mathrm{mTc}-W B C$ SPECT/CT to monitor the effect of therapy in diabetic foot osteomyelitis. Int Wound J 13:1158-1160

27. Filippi L, Uccioli L, Giurato L, Schillaci O (2009) Diabetic foot infection: usefulness of SPECT/CT for $99 \mathrm{mTc}-\mathrm{HMPAO}$-labeled leukocyte imaging. J Nucl Med 50:1042-1046
28. La Fontaine J, Bhavan K, Lam K et al (2016) Comparison between Tc-99m WBC SPECT/CT and MRI for the diagnosis of biopsyproven diabetic foot osteomyelitis. Wounds 28:271-278

29. Saeed S, Zafar J, Khan B et al (2013) Utility of ${ }^{99} \mathrm{mTc}$-labelled antimicrobial peptide ubiquicidin (29-41) in the diagnosis of diabetic foot infection. Eur J Nucl Med Mol Imaging 40:737-743

30. Aslangul E, M'bemba J, Caillat-Vigneron N et al (2013) Diagnosing diabetic foot osteomyelitis in patients without signs of soft tissue infection by coupling hybrid $67 \mathrm{Ga}$ SPECT/CT with bedside percutaneous bone puncture. Diabetes Care 36:2203-2210

31. Rastogi A, Bhattacharya A, Prakash M et al (2016) Utility of PET/ CT with fluorine-18-fluorodeoxyglucose-labeled autologous leukocytes for diagnosing diabetic foot osteomyelitis in patients with Charcot's neuroarthropathy. Nucl Med Commun 37:1253-1259

32. Zuluaga AF, Galvis W, Jaimes F, Vespa O (2002) Lack of microbiological concordance between bone and non-bone specimens in chronic osteomyelitis: an observational study. BMC Infect Dis 16:2-8

33. Senneville E, Morant H, Descamps D et al (2009) Needle puncture and transcutaneous bone biopsy cultures are inconsistent in patients with diabetes and suspected osteomyelitis of the foot. Clin Infect Dis 48:888-893

34. Poirier JY, Garin E, Derrien C et al (2002) Diagnosis of osteomyelitis in the diabetic foot with a 99mTc-HMPAO leucocyte scintigraphy combined with a $99 \mathrm{mTc}-\mathrm{MDP}$ bone scintigraphy. Diabetes Metab 28:485-490

35. Filippi L, Schillaci O (2006) Usefulness of hybrid SPECT/CT in 99mTc-HMPAO-labeled leukocyte scintigraphy for bone and joint infections. J Nucl Med 47:1908-1913

36. Ballani NS, Al-Huda FA, Khan HA et al (2007) The value of quantitative uptake of (99m)Tc-MDP and (99m)Tc-HMPAO white blood cells in detecting osteomyelitis in violated peripheral bones. J Nucl Med Technol 35:91-95

37. Datz FL, Seabold JE, Brown ML et al (1997) Procedure guideline for technetium-99m exametazime (HMPAO) labeled leukocyte scintigraphy for suspected infection/inflammation. J Nucl Med 38:987-990 